\title{
Improved Stability and Stabilization Results for Discrete Singular Delay Systems via Delay Partitioning
}

Zhiguang Feng James Lam Huijun Gao Baozhu Du

on continuous-time systems [7], discrete-time cases [16]

\begin{abstract}
In this paper, the problems of delay-dependent robust stability analysis and robust stabilization are investigated for uncertain discrete-time singular systems with state delay. First, by making use of the delay partitioning technique, a new delay-dependent criterion is given to ensure the nominal system to be regular, causal and stable. This new criterion is further extended to singular systems with both delay and parameter uncertainties. Moreover, without the assumption that the considered systems being regular and causal, robust controllers are designed for discrete-time singular time-delay systems such that the closed-loop systems have the characteristics of regularity, causality and asymptotic stability. These results are illustrated, via a few numerical examples, to be much less conservative than most of the existing results in the literature.
\end{abstract}

\section{INTRODUCTION}

Singular systems, also called descriptor systems, semistate-space systems and generalized state-space systems, frequently appear in various engineering systems, such as aircraft attitude control systems, flexible robots, large-scale electric networks, chemical engineering systems, lossless transmission lines [10]. Such systems provide a more natural description of dynamic systems than the standard state-space systems due to the fact that the singular systems can preserve the structure of physical systems more accurately by including non-dynamic constraints and impulsive elements. On the other hand, time delays frequently induce instability and are commonly regarded as one of the main factors that degrade system performance. Hence, there are a great number of research results concerning time-delay systems [1], [8], [2], [14]. Singular time-delay systems are in essence delay differential equations coupled with functional equations, and thus the robust stability problem for singular systems is much more complicated than that for state-space systems because it requires to consider not only stability robustness, but also regularity and causality (absence of impulses) which may affect the stability of the system. The problems arising from singular time-delay systems are significant both in theory and in practice. A considerable number of studies have been devoted to singular time-delay systems, such as the results

This work was partially supported by the Research Fund for the Doctoral Program of Higher Education of China (20070213084), the Fok Ying Tung Education Foundation (111064), the Foundation for the Author of National Excellent Doctoral Dissertation of China (2007B4), and HKU RGC Grant 7029/05P.

J. Lam, Z. Feng and B. Du are with Department of Mechanical Engineering, The University of Hong Kong, Pokfulam Road, Hong Kong. Email: james.lam@hku.hk, congdian@gmail.com

H. Gao is with Space Control and Inertial Technology Research Center, Harbin Institute of Technology, Harbin, Heilongjiang Province, 150001, China. Email: hjgao@hit.edu.cn and the references therein.

As problems of fundamental importance, stability analysis and synthesis have been on the forefront of the research on time-delay singular systems and a great number of results based on the theory of state-space systems have been extended to singular systems [4]. In the continuous context, the robust stability and robust stabilization problems are solved based on the concepts of generalized quadratic stability and generalized quadratic stabilization [19]. While the results are delay-independent, and thus quite conservative especially when the delay is comparatively small. Thus, considerable efforts are devoted to establish delay-dependent conditions. In [20], a delay-dependent robust stability criterion is proposed and the problem of robust stabilization is addressed for singular time-delay systems with normbounded parameter uncertainty which improves the results in [19] to a certain extent. Furthermore, even less conservative result is obtained in [9] by avoiding an upper bounding on the weighted cross products of the state and the delayed state. [7] presents an LMI approach to singular systems with state delay by utilizing decomposing system technique and the results are in terms of the coefficient matrices of the decomposed systems. While [18] provides a new delaydependent bound real lemma avoiding some computational problems arising from decomposition of the original singular systems and further reduces the conservatism in [7]. In the discrete setting, the robust stability problem is studied in [11] which presents the results in terms of strict linear matrix inequalities (LMIs) making the verification procedure relatively simple and reliable. By introducing a finite sum inequality, [15] presents less conservative results than those in [11] without any additional assumption on the system matrices. It should be pointed out that the results formulated above make significant contributions to the development of the singular system theory. However, to the best of the authors' knowledge, the analysis and synthesis problems for discrete singular time-delay systems with parametric uncertainties have not been fully investigated yet and the results reported in the literature still leave much room for improvement which motivates the present study.

In this paper we consider the problems of delay-dependent stability analysis and stabilization for linear discrete-time uncertain singular systems with state delay. With the introduction of the delay partitioning technique, strict LMI sufficient criteria are obtained for discrete-time singular systems to be regular, causal, and stable. Based on these criteria, the robust stabilization problem is addressed and an 
explicit expression of the desired state-feedback controllers are given. Numerical examples are given to illustrate the reduction of conservatism of the developed results.

Notation: The notation used throughout the paper is fairly standard. $\mathbb{R}^{n}$ denotes the $n$-dimensional Euclidean space and $P>0(\geq 0)$ means that $P$ is real symmetric and positive definite (semi-definite); $I$ and 0 refer to the identity matrix and zero matrix with compatible dimensions; « stands for the symmetric terms in a symmetric matrix and $\operatorname{sym}(A)$ is defined as $A+A^{T}$; $\bullet$ represents matrices that are not relevant with our discussion. Matrices with the same letters refer to the same definitions and matrices are assumed to be compatible for algebraic operations if their dimensions are not explicitly stated.

\section{Problem Formulation}

Consider a class of linear discrete-time uncertain singular systems with state delay described by

$$
\left\{\begin{array}{c}
\operatorname{Ex}(k+1)=(A+\Delta A) x(k)+\left(A_{d}+\Delta A_{d}\right) x(k-d) \\
+(B+\Delta B) u(k), \\
x(k)=\phi(k), k \in[-\bar{d}, 0],
\end{array}\right.
$$

where $x(k) \in \mathbb{R}^{n}$ is the state vector; $u(k) \in \mathbb{R}^{q}$ is the control input; $A, A_{d}$ and $B$ denote constant matrices with appropriate dimensions; $d$ is a constant positive integer satisfying $0<$ $d \leq \bar{d}$ ( $d$ can always be described by $d=m \tau$, where $m$ and $\tau$ are integers), where $\bar{d}$ is a positive integer representing the upper bound of the delay; matrix $E$ may be singular and rank $E=r \leq n ; \phi(k)$ is a compatible vector valued initial function; $\Delta A, \Delta A_{d}$ and $\Delta B$ are time-varying uncertain matrices of the form

$$
\left[\begin{array}{lll}
\Delta A & \Delta A_{d} & \Delta B
\end{array}\right]=\operatorname{MF}(k)\left[\begin{array}{lll}
N_{1} & N_{2} & N_{3}
\end{array}\right],
$$

where $M, N_{1}, N_{2}$ and $N_{3}$ are constant matrices, and $F(k) \in$ $\mathbb{R}^{l \times b}$ is an unknown real matrix satisfying $F(k) F(k)^{T} \leq I$.

Before moving on, we give some definitions and lemmas concerning the following nominal unforced counterpart of the system in (1):

$$
\left\{\begin{array}{c}
E x(k+1)=A x(k)+A_{d} x(k-d), \\
x(k)=\phi(k), k \in[-\bar{d}, 0] .
\end{array}\right.
$$

\section{Definition 1: [4]}

1) The pair $(E, A)$ is regular, if $\operatorname{det}(z E-A)$ is not identically zero.

2) The pair $(E, A)$ is said to be causal, if it is regular and $\operatorname{deg}\{\operatorname{det}(z E-A)\}=\operatorname{rank} E$.

Lemma 1: [11] Suppose the pair $(E, A)$ is regular and causal, then the solution to system (2) is causal and unique on $[0, \infty)$ for any constant time delay $d$ satisfying $0<d \leq \bar{d}$.

In view of this, we introduce the following definition for singular delay system (2).

\section{Definition 2:}

1) The singular delay system in (2) is said to be regular and causal if the pair $(E, A)$ is regular and causal.

2) The singular system in (2) is said to be asymptotically stable, if for any $\varepsilon>0$, there exists a scalar $\delta(\varepsilon)>0$, such that for any compatible initial conditions $\phi(k)$ satisfying $\sup _{-\bar{d} \leq k \leq-1}\|\phi(k)\| \leq \delta(\varepsilon)$, the solution $x(k)$ of (2) satisfies $\|x(k)\| \leq \varepsilon$ for $k \geq 0$; furthermore, $x(k) \rightarrow 0$, when $k \rightarrow$ $\infty$.

3) The discrete singular time-delay system in (2) is said to be admissible if it is regular, causal and asymptotically stable.

Lemma 2: [13] The system in (2) is asymptotically stable if and only if $\operatorname{det}\left(z E-A-z^{-d} A_{d}\right) \neq 0$ for $|z| \geq 1$.

Lemma 3: [12] Given matrices $\Omega, \Gamma$ and $\Phi$ with appropriate dimensions and with $\Omega$ symmetrical, then

$$
\Omega+\Gamma F \Phi+\Phi^{T} F^{T} \Gamma^{T}<0
$$

for any $F$ satisfying $F^{T} F \leq I$, if and only if there exists a scalar $\varepsilon>0$ such that

$$
\Omega+\varepsilon \Gamma \Gamma^{T}+\varepsilon^{-1} \Phi^{T} \Phi<0 .
$$

The objective of this paper is to establish new robust stability criteria such that the unforced discrete-time singular system of (1) is admissible and to develop a procedure to design stabilizing state-feedback controllers for the uncertain discrete system in (1) such that the resulting closed-loop system is admissible.

\section{MAin Results}

In this section, we obtain a solution to the robust stability analysis and robust stabilization problems formulated previously by using a strict LMI approach. First, we present the following result for the nominal singular delay systems, which will play a key role in solving the aforementioned problems.

\section{A. Stability Analysis: Nominal Case}

In this subsection, we will present a new delay-dependent sufficient condition guaranteeing the nominal system in (2) is admissible.

Theorem 1: Given positive integers $m, \tau$, the system in (2) is admissible, if there exist matrices $P_{1}>0, Q>0, Z>0$, $Y_{1}, Y_{2}, T_{1}, S_{1}, S_{2}, S_{3}, P_{2}, P_{3}$, and $P_{4}$, such that

$$
\left[\begin{array}{cc}
\Theta & \tau Y \\
\star & -\tau Z
\end{array}\right]<0
$$

where $R \in \mathbb{R}^{n \times(n-r)}$ is any full-column rank matrix satisfying 
$E^{T} R=0$ and

$$
\begin{aligned}
& \Theta=W_{P}^{T}\left(P_{1}+\tau Z\right) W_{P}+W_{Q}^{T} \tilde{Q} W_{Q} \\
& +\operatorname{sym}\left(W_{P 1}^{T} E^{T} P_{1} W_{P}+P^{T} W_{P 2}+S R^{T} W_{P}+Y E W_{Y}\right), \\
& \tilde{Q}=\left[\begin{array}{cc}
Q & 0_{m n, m n} \\
0_{m n, m n} & -Q
\end{array}\right], Q=\left[\begin{array}{ccc}
Q_{11} & \cdots & Q_{1 m} \\
\star & \ddots & \vdots \\
\star & \star & Q_{m m}
\end{array}\right] \text {, } \\
& S=\left[\begin{array}{llll}
S_{1}^{T} & S_{2}^{T} & 0_{n-r,(m-1) n} & S_{3}^{T}
\end{array}\right]^{T}, \\
& W_{Y}=\left[\begin{array}{lll}
I_{n} & -I_{n} & 0_{n, m n}
\end{array}\right], \\
& W_{P 1}=\left[\begin{array}{ll}
I_{n} & 0_{n,(m+1) n}
\end{array}\right], W_{P}=\left[\begin{array}{ll}
0_{n,(m+1) n} & I_{n}
\end{array}\right] \text {, } \\
& P=\left[\begin{array}{llll}
P_{2} & P_{4} & 0_{n,(m-1) n} & P_{3}
\end{array}\right], \\
& Y^{T}=\left[\begin{array}{lll}
Y_{1}^{T} & T_{1}^{T} & Y_{2}^{T}
\end{array}\right], \\
& Y_{1}=\left[\begin{array}{llll}
Y_{1 y}^{T} & Y_{2 y}^{T} & \cdots & Y_{m y}^{T}
\end{array}\right]^{T},
\end{aligned}
$$

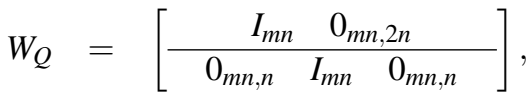

$$
\begin{aligned}
& W_{P 2}=\left[\begin{array}{llll}
A-E & 0_{n,(m-1) n} & A_{d} & -I_{n}
\end{array}\right] .
\end{aligned}
$$

Proof. First, we prove the regularity and causality of the system. Let

$$
\begin{aligned}
\bar{E} & =\left[\begin{array}{cc}
E & 0 \\
0 & 0
\end{array}\right], \bar{A}=\left[\begin{array}{cc}
E & I \\
A-E & -I
\end{array}\right], \\
\bar{P} & =\left[\begin{array}{cc}
P_{1} & 0 \\
0 & 0
\end{array}\right], \bar{Y}=\left[\begin{array}{cc}
Y_{1 y} & 0 \\
Y_{2} & 0
\end{array}\right], \bar{T}=\left[\begin{array}{cc}
T_{1} & 0 \\
0 & 0
\end{array}\right], \\
\bar{Q} & =\left[\begin{array}{cc}
Q_{11} & 0 \\
0 & \tau Z
\end{array}\right], \bar{S}=\left[\begin{array}{ll}
S_{1} & P_{2}^{T} \\
S_{3} & P_{3}^{T}
\end{array}\right], \bar{R}=\left[\begin{array}{ll}
R & 0 \\
0 & I
\end{array}\right] .
\end{aligned}
$$

Since $\operatorname{rank} \bar{E}=\operatorname{rank} E=r \leq n$, there exist nonsingular matrices $U$ and $V$, such that

$$
U \bar{E} V=\left[\begin{array}{cc}
I_{r} & 0 \\
0 & 0
\end{array}\right] .
$$

Denote

$$
\begin{aligned}
U \bar{A} V & =\left[\begin{array}{ll}
A_{11} & A_{12} \\
A_{21} & A_{22}
\end{array}\right], U^{-T} \bar{P} U^{-1}=\left[\begin{array}{ll}
P_{11} & P_{12} \\
P_{21} & P_{22}
\end{array}\right], \\
V^{T} \bar{Y} U^{-1} & =\left[\begin{array}{ll}
Y_{11} & Y_{12} \\
Y_{21} & Y_{22}
\end{array}\right], V^{T} \bar{S}=\left[\begin{array}{l}
S_{11} \\
S_{21}
\end{array}\right], \\
U^{-T} \bar{R} & =\left[\begin{array}{l}
0 \\
I
\end{array}\right] H,
\end{aligned}
$$

where $H \in \mathbb{R}^{(2 n-r) \times(2 n-r)}$ is a nonsingular matrix determined by $U^{-T} \bar{R}$. From (3), it is easy to see that

$$
\Theta<0
$$

Define

$$
L=\left[\begin{array}{cccc}
I_{n} & 0 & 0_{n,(m-1) n} & 0 \\
0 & 0 & 0_{n,(m-1) n} & I_{n} \\
0_{(m-1) n, n} & 0_{(m-1) n, n} & I_{(m-1) n} & 0_{(m-1) n, n} \\
0 & I_{n} & 0 & 0
\end{array}\right] .
$$

Then performing a congruence transformation to (4) by $L$, we obtain the following inequality

$$
\left[\begin{array}{cccc}
\Theta_{11} & \Theta_{13} & \bullet & \bullet \\
\star & \Theta_{33} & \bullet & \bullet \\
\bullet & \bullet & \bullet & \bullet \\
\bullet & \bullet & \bullet & \bullet
\end{array}\right]<0
$$

where

$$
\begin{aligned}
& \Theta_{11}=P_{2}^{T}(A-E)+(A-E)^{T} P_{2}+Y_{1 y} E+E^{T} Y_{1 y}^{T}+Q_{11}, \\
& \Theta_{13}=-P_{2}^{T}+(A-E)^{T} P_{3}+S_{1} R^{T}+E^{T} Y_{2}^{T}+E^{T} P_{1}, \\
& \Theta_{33}=\tau Z+P_{1}-P_{3}-P_{3}^{T}+S_{3} R^{T}+R S_{3}^{T} .
\end{aligned}
$$

From (5), we have

$$
\bar{A}^{T} \bar{P} \bar{A}-\bar{E}^{T} \bar{P} \bar{E}+\bar{S} \bar{R}^{T} \bar{A}+\bar{A}^{T} \bar{R} \bar{S}^{T}+\bar{Y} \bar{E}+\bar{E}^{T} \bar{Y}^{T}+\bar{Q}<0,
$$

which implies that

$$
-\bar{E}^{T} \bar{P} \bar{E}+\bar{S} \bar{R}^{T} \bar{A}+\bar{A}^{T} \bar{R} \bar{S}^{T}+\bar{Y} \bar{E}+\bar{E}^{T} \bar{Y}^{T}<0 .
$$

Performing a congruence transformation to (6) by $V^{T}$ and $V$, we obtain

$$
\left[\begin{array}{lc}
\bullet & \bullet \\
\bullet & S_{21} H^{T} A_{22}+A_{22}^{T} H S_{21}^{T}
\end{array}\right]<0,
$$

which implies that $A_{22}$ is nonsingular. Hence,

$$
\begin{aligned}
\operatorname{det}(z \bar{E}-\bar{A})= & \operatorname{det}\left(U^{-1}\right) \operatorname{det}\left(z I_{r}-A_{11}+A_{12} A_{22}^{-1} A_{21}\right) \\
& \times \operatorname{det}\left(-A_{22}\right) \operatorname{det}\left(V^{-1}\right)
\end{aligned}
$$

is not identically zero and $\operatorname{deg} \operatorname{det}(z \bar{E}-\bar{A})=r$. This together with Definition 1 leads to that the pair $(\bar{E}, \bar{A})$ is regular and causal. Noticing the fact that

$$
\begin{aligned}
\operatorname{det}(z E-A) & =\operatorname{det}(z \bar{E}-\bar{A}), \\
\operatorname{deg}(\operatorname{det}(z E-A)) & =\operatorname{deg}(\operatorname{det}(z \bar{E}-\bar{A})),
\end{aligned}
$$

we can easily see that the pair $(E, A)$ is regular and causal. Then according to Lemma 1 and Definition 2, the system in (2) is regular and causal.

Then we are in position to show that system (2) is asymptotically stable. To this end, we choose a new Lyapunov functional candidate as

$$
V(k)=V_{1}(k)+V_{2}(k)+V_{3}(k),
$$

where

$$
\begin{aligned}
V_{1}(k) & =x^{T}(k) E^{T} P_{1} E x(k), \\
V_{2}(k) & =\sum_{i=k-\tau}^{k-1} \Upsilon^{T}(i) Q \Upsilon(i), \\
V_{3}(k) & =\sum_{i=-\tau}^{-1} \sum_{j=k+i}^{k-1} \eta^{T}(j) Z \eta(j),
\end{aligned}
$$

and

$$
\Upsilon(i)=\left[\begin{array}{c}
x(i) \\
x(i-\tau) \\
x(i-2 \tau) \\
\vdots \\
x(i-\tau m+\tau)
\end{array}\right], \eta(j)=\operatorname{Ex}(j+1)-\operatorname{Ex}(j)
$$

Taking the forward difference of the functional in (7) along the solution of system (2), and defining

$$
\xi(k)=\left[\begin{array}{c}
\Upsilon(k) \\
x(k-m \tau) \\
\eta(k)
\end{array}\right],
$$


we have

$$
\begin{aligned}
\Delta V_{1}(k)= & x^{T}(k+1) E^{T} P_{1} E x(k+1)-x^{T}(k) E^{T} P_{1} E x(k) \\
= & {[E x(k+1)-E x(k)]^{T} P_{1}[E x(k+1)-E x(k)] } \\
& +2 x(k)^{T} E^{T} P_{1}[E x(k+1)-E x(k)] \\
& +2\left[x(k)^{T} P_{2}^{T}+[E x(k+1)-E x(k)]^{T} P_{3}^{T}\right. \\
& \left.+x(k-\tau)^{T} P_{4}^{T}\right]\left[(A-E) x(k)+A_{d} x(k-m \tau)\right. \\
& -[E x(k+1)-E x(k)]]+2\left[x(k)^{T} S_{1} R^{T}\right. \\
& \left.+x(k-\tau)^{T} S_{2} R^{T}+[E x(k+1)-E x(k)]^{T} S_{3} R^{T}\right] \\
& \times[E x(k+1)-E x(k)] \\
= & \xi^{T}(k) W_{P}^{T} P_{1} W_{P} \xi(k)+2 \xi^{T}(k)\left(W_{P 1}^{T} E^{T} P_{1} W_{P}\right. \\
& \left.+P^{T} W_{P 2}+S R^{T} W_{P}\right) \xi(k), \\
\Delta V_{2}(k)= & \Upsilon^{T}(k) Q \Upsilon(k)-\Upsilon^{T}(k-\tau) Q \Upsilon(k-\tau) \\
= & \xi^{T}(k) W_{Q}^{T} \tilde{Q} W_{Q} \xi(k), \\
\Delta V_{3}(k)= & \tau \eta^{T}(k) Z \eta(k)-\sum_{i=k-\tau}^{k-1} \eta^{T}(i) Z \eta(i) \\
\leq & \tau \eta^{T}(k) Z \eta(k)+\tau \xi(k)^{T} Y Z^{-1} Y^{T} \xi(k) \\
& +2 \xi(k)^{T} Y E[x(k)-x(k-\tau)]
\end{aligned}
$$

By connecting (8)-(10), we obtain

$$
\Delta V(k) \leq \xi(k)^{T}\left(\Theta+\tau Y Z^{-1} Y^{T}\right) \xi(k) .
$$

By using Schur complement, (3) implies $\Theta+\tau Y Z^{-1} Y^{T}<$ 0 , Then from Definition 2, we conclude that the system is asymptotically stable and this completes the proof.

Remark 1: The main technique utilized in this paper is the delay partitioning idea which partitions the time delay for $m$ times. When $m=1$, the results obtained in Theorem 2 is equivalent to Theorem 1 in [15].

\section{B. Stabilization: Nominal Case}

Based on Theorem 2, a state-feedback controller

$$
u(k)=K x(k), K \in \mathbb{R}^{q \times n},
$$

will be designed for the nominal singular system such that the resultant closed-loop system is admissible. The controller results in the following closed-loop system

$$
E x(k+1)=(A+B K) x(k)+A_{d} x(k-d) .
$$

Then, we have the following result.

Theorem 2: Given scalars $\gamma_{1}, \gamma_{2}, \gamma_{3}$ and positive integers $m, \tau$, there exists a state-feedback controller in the form of (12) such that the closed-loop system in (13) is admissible if there exist matrices $P_{1}>0, Q>0, Z>0, Y_{1}, Y_{2}, T_{1}, S_{1}$, $S_{2}, S_{3}, J, X$, such that

$$
\left[\begin{array}{cc}
\Psi & \tau Y \\
\star & -\tau Z
\end{array}\right]<0
$$

where

$$
\begin{aligned}
\Psi= & W_{P}^{T}\left(P_{1}+\tau Z\right) W_{P}+W_{Q}^{T} \tilde{Q} W_{Q} \\
& +\operatorname{sym}\left(W_{P 1}^{T} E P_{1} W_{P}+W_{E}^{T} \Lambda+S R^{T} W_{P}+Y E^{T} W_{Y}\right), \\
W_{E}= & {\left[\begin{array}{llll}
\gamma_{1} I_{n} & \gamma_{3} I_{n} & 0_{n,(m-1) n} & \gamma_{2} I_{n}
\end{array}\right], } \\
\Lambda= & {\left[\begin{array}{llll}
J^{T}(A-E)^{T}+X^{T} B^{T} & 0_{n,(m-1) n} & J^{T} A_{d}^{T} & -J^{T}
\end{array}\right] . }
\end{aligned}
$$

Moreover, if the above condition is feasible, a desired controller gain matrix in the form of (12) is given by

Proof. It is easy to see that

$$
K=X J^{-1} \text {. }
$$

$$
\operatorname{det}(z E-(A+B K))=\operatorname{det}\left(z E^{T}-(A+B K)^{T}\right),
$$

$\operatorname{deg}(\operatorname{det}(z E-(A+B K)))=\operatorname{deg}\left(\operatorname{det}\left(z E^{T}-(A+B K)^{T}\right)\right)$,

and that $\operatorname{det}\left(z E-(A+B K)-z^{-d} A_{d}\right)=0$ and $\operatorname{det}\left(z E^{T}-(A+\right.$ $\left.B K)^{T}-z^{-d} A_{d}^{T}\right)=0$ have the same solution set. With respect to the regularity, causality and stability of a system, we obtain that the system in (13) is equivalent to the following system based on Definition 1 and Lemma 2,

$$
E^{T} \delta(k+1)=(A+B K)^{T} \delta(k)+A_{d}^{T} \delta(k-d) .
$$

Substituting $E, A$, and $A_{d}$ with $E^{T},(A+B K)^{T}$ and $A_{d}^{T}$ in (3), respectively, we have the following inequality

$$
\left[\begin{array}{cc}
\Psi_{1} & \tau Y \\
\star & -\tau Z
\end{array}\right]<0
$$

where

$$
\begin{aligned}
\Psi_{1}= & W_{P}^{T}\left(P_{1}+\tau Z\right) W_{P}+W_{Q}^{T} \tilde{Q} W_{Q} \\
& +\operatorname{sym}\left(W_{P 1}^{T} E P_{1} W_{P}+S R^{T} W_{P}+Y E^{T} W_{Y}\right. \\
& +\left[\begin{array}{llll}
P_{2} & P_{4} & 0_{n,(m-1) n} & P_{3}
\end{array}\right]^{T} \\
& \left.\times\left[\begin{array}{llll}
(A-E+B K)^{T} & 0_{n,(m-1) n} & A_{d}^{T} & -I_{n}
\end{array}\right]\right) .
\end{aligned}
$$

Then, by denoting $P_{2}=\gamma_{1} J, P_{3}=\gamma_{2} J, P_{4}=\gamma_{3} J$, and $X=K J$, (14) is readily obtained and theorem is proved.

\section{Uncertain Case}

In this subsection, the problems of robust stability analysis and stabilization for the uncertain singular system in (1) are considered. For system (1) with time-varying structured uncertainties, we have the following theorem.

Theorem 3: Given positive integers $m, \tau$, the time-delay system in (1) with $u(t)=0$ is admissible for all parameter uncertainties if there exist matrices $P_{1}>0, Q>0, Z>0, Y_{1}$, $Y_{2}, T_{1}, S_{1}, S_{2}, S_{3}, P_{2}, P_{3}, P_{4}$, and a scalar $\varepsilon>0$, such that

$$
\left[\begin{array}{ccc}
\Theta+\varepsilon \Xi^{T} \Xi & \tau Y & P^{T} M \\
\star & -\tau Z & 0 \\
\star & \star & -\varepsilon I
\end{array}\right]<0,
$$

where

$$
\Xi=\left[\begin{array}{llll}
N_{1} & 0_{b,(m-1) n} & N_{2} & 0_{b, n}
\end{array}\right] .
$$

Proof. Based on Theorem 2, by replacing $A$ and $A_{d}$ in (3) with $A+M F(k) N_{1}$ and $A_{d}+M F(k) N_{2}$, respectively, the stability criterion for the uncertain system can be rewritten as

$$
\left[\begin{array}{cc}
\Theta+\operatorname{sym}\left(P^{T} M F(k) \Xi\right) & \tau Y \\
\star & -\tau Z
\end{array}\right]<0 .
$$

Applying the Schur complement to (15), then we can obtain (16) by Lemma 3 and the proof is completed.

Next, we will consider the robust controller design for the system in (1) with $u(k)=K x(k)$ such that the closedloop system is admissible for all parameter uncertainties. 
Applying the inequality in (14) to the uncertain singular system in (1), we can easily obtain the following theorem.

Theorem 4: Given scalars $\gamma_{1}, \gamma_{2}, \gamma_{3}$ and positive integers $m, \tau$, there exists a state-feedback controller in the form of (12) such that the closed-loop system in (1) is admissible if there exist matrices $P_{1}>0, Q>0, Z>0, Y_{1}, Y_{2}, T_{1}, S_{1}, S_{2}$, $S_{3}, J, X$, and a positive scalar $\gamma$,such that

$$
\left[\begin{array}{cccc}
\Psi+\gamma \Gamma \Gamma^{T} & \tau Y & \Phi_{1}^{T} & \Phi_{2}^{T} \\
\star & -\tau Z & 0_{n, n} & 0_{n, n} \\
\star & \star & -\gamma & 0_{n, n} \\
\star & \star & \star & -\gamma
\end{array}\right]<0,
$$

where

$$
\begin{aligned}
\Phi_{1}= & {\left[\begin{array}{lll}
\gamma_{1}\left(N_{1} J+N_{3} X\right) & \gamma_{3}\left(N_{1} J+N_{3} X\right) \\
0_{b,(m-1) n} & \gamma_{2}\left(N_{1} J+N_{3} X\right)
\end{array}\right], } \\
\Phi_{2}= & {\left[\begin{array}{llll}
\gamma_{1} N_{2} J & \gamma_{3} N_{2} J & 0_{b,(m-1) n} & \gamma_{2} N_{2} J
\end{array}\right], } \\
\Gamma^{T=} & {\left[\begin{array}{llll}
M^{T} & 0_{l,(m-1) n} & 0_{l, n} & 0_{l, n} \\
0_{l, n} & 0_{l,(m-1) n} & M^{T} & 0_{l, n}
\end{array}\right] . }
\end{aligned}
$$

Moreover, if the above condition is feasible, a desired controller gain matrix in the form of (12) is given by

$$
K=X J^{-1} \text {. }
$$

Remark 2: In order to establish the stability conditions for the singular systems with constant delay, the considered systems are converted to delay-free systems by the state augmentation approach in [17], [16]. However, the order of the transformed systems is high if the delay is large and the method becomes difficult to apply for unknown delay or time-varying delay cases. On the other hand, although the state augmentation method might be good for stability analysis as it may provide necessary and sufficient conditions, it does not provide an easy way for controller design problem.

Remark 3: The reduced conservatism of the above results benefits from the construction of the new Lyapunov functional candidate in (7) by utilizing the delay partitioning technique. This reduced conservatism is more prominent when the partitioning number $m$ increases. In addition the delay partitioning technique has also been applied to stability analysis of continuous systems with multiple delay components in [5] and neutral delay systems in [6].

\section{ILlustrative EXAMPLES}

Example 1: Consider the singular system with the following parameters

$$
\left[\begin{array}{cc}
3.5 & 0 \\
0 & 0
\end{array}\right] x(k+1)=\left[\begin{array}{cc}
a_{11} & 0 \\
0 & -3
\end{array}\right] x(k)+\left[\begin{array}{cc}
-1.3 & 1.5 \\
0 & 0.5
\end{array}\right] x(k-d) \text {. }
$$

Our purpose is to determine the allowable time delay upper bounds $\bar{d}$ such that the system is admissible. To compare our results with those in [11], [15], we consider $a_{11}=2.3$, or $a_{11}=2.4$. Table 1 gives more detailed comparison results on the maximum allowed bounds for $\bar{d}$ via the methods in [11], [15] and Theorem 1 in this paper. The results in Table 1 clearly show that the result in this paper outperforms those in [11], [15] in terms of conservatism.
Next, the advantages of our results will be shown by considering an uncertain discrete-time singular delay system in Example 2.

Example 2: Consider the uncertain discrete-time singular system with the following parameters (borrowed from [15])

$$
\begin{aligned}
{\left[\begin{array}{ll}
2 & 0 \\
0 & 0
\end{array}\right] x(k+1)=} & {\left[\begin{array}{cc}
0.9977+0.1 \alpha & 1.1972 \\
0.1001 & -1.9
\end{array}\right] x(k) } \\
& +\left[\begin{array}{cc}
-1.1972 & 1.5772 \\
0 & 0.9757+0.1 \alpha
\end{array}\right] x(k-d) .
\end{aligned}
$$

The purpose is to determine the upper bounds for the absolute value of uncertain parameter $\alpha$, that is $\bar{\alpha}$. To illustrate the benefits of our results, Table 2 gives the comparison results on $\bar{\alpha}$.

These comparison results show that the result in Theorem 3 for delay singular systems with uncertainties in this paper is less conservative than those in [11], [15].

In Example 3, the applicability of the proposed controller design methods will be demonstrated.

Example 3: Consider the uncertain singular system in (1) with the following parameters:

$$
\begin{aligned}
E & =\left[\begin{array}{ll}
1 & 0 \\
0 & 0
\end{array}\right], A=\left[\begin{array}{cc}
1.7 & 2 \\
1 & 2
\end{array}\right], A_{d}=\left[\begin{array}{cc}
1.5 & 1 \\
1 & 0.05
\end{array}\right], \\
B & =\left[\begin{array}{cc}
-2 & 3 \\
0 & -2
\end{array}\right], M=\left[\begin{array}{c}
0.2 \\
0.2
\end{array}\right], F(k)=\sin (k), \\
N_{1} & =N_{2}=\left[\begin{array}{ll}
0.2 & 0.2
\end{array}\right], N_{3}=\left[\begin{array}{ll}
0.01 & 0.02
\end{array}\right] .
\end{aligned}
$$

In this example, we choose that

$$
\gamma_{1}=0.6, \gamma_{2}=1, \gamma_{3}=-0.35, R=\left[\begin{array}{ll}
0 & 1
\end{array}\right]^{T}, d=3 .
$$

Therefore, by Theorem 4, an admissible state-feedback control law can be obtained by solving the LMIs in (17):

$$
u(k)=\left[\begin{array}{rr}
-2.9773 & -0.5313 \\
-2.3906 & -0.9896
\end{array}\right] x(k)
$$

Figure 1 and Figure 2 give the simulation results of two states with and without the state-feedback control, respectively. From Figure 1 and Figure 2, we can see that the open-loop system is unstable and the closed-loop system is stable.

\section{Conclusions}

In this paper, improved functionals based on the delay partitioning technique have been introduced to derive improved results for robust stability and stabilization of linear uncertain discrete-time singular systems with state delay. The resulting criteria have been formulated in terms of strict LMIs involving no decomposition of the system matrices. Numerical examples have been given to demonstrate the advantages and the merits of the proposed results. 
FrAln4.12

Table 1 Comparisons of maximum allowed delay $\bar{d}$

\begin{tabular}{ccc}
\hline$a_{11}$ & 2.3 & 2.4 \\
\hline $\bar{d}[11]$ & 10 & 7 \\
\hline $\bar{d}[15]$ & 11 & 7 \\
\hline $\bar{d}$ (Theorem 1) & $11(m=1, \tau=11)$ & $7(m=1, \tau=7)$ \\
\hline $\bar{d}$ (Theorem 1) & $12(m=3, \tau=4)$ & $9(m=3, \tau=3)$ \\
\hline $\bar{d}$ (Theorem 1$)$ & $14(m=7, \tau=2)$ & $10(m=5, \tau=2)$ \\
\hline
\end{tabular}

Table 2 Allowable maximum absolute value of $\alpha$ obtained by different methods

\begin{tabular}{llll}
\hline $\bar{d}$ & 2 & 3 & 4 \\
\hline $\bar{\alpha}[11]$ & 1.9464 & 0.8033 & 0.1563 \\
\hline $\bar{\alpha}[15]$ & 2.1359 & 1.0325 & 0.2853 \\
\hline $\bar{\alpha}($ Theorem 3$)$ & $2.1359(m=1, \tau=2)$ & $1.0325(m=1, \tau=3)$ & $0.2853(m=1, \tau=4)$ \\
\hline $\bar{\alpha}($ Theorem 3$)$ & $2.6847(m=2, \tau=1)$ & $1.6734(m=3, \tau=1)$ & $0.8972(m=4, \tau=1)$ \\
\hline
\end{tabular}

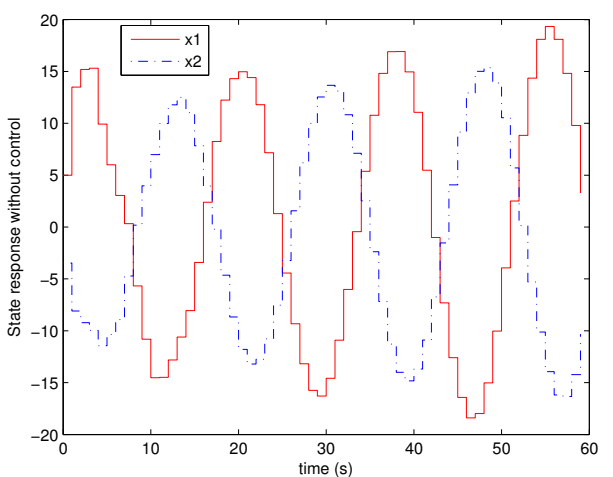

Fig. 1. The state trajectories of the open-loop system

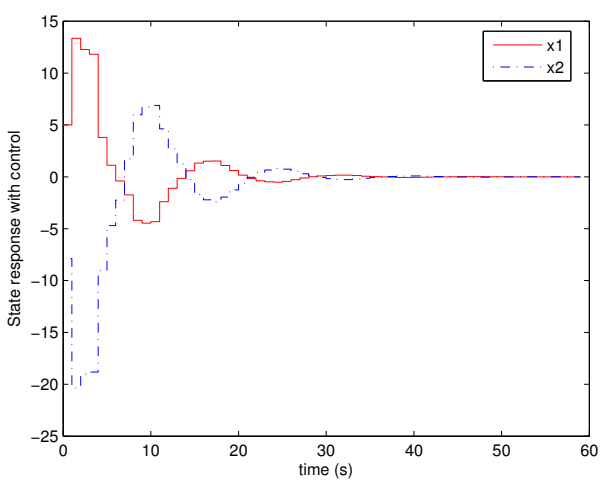

Fig. 2. The state trajectories of the closed-loop system

\section{REFERENCES}

[1] K. Gu, V.L.Kharitonov, and J. Chen. Stability of Time-Delay Systems. Springer-Verlag, Berlin, Germany, 2003.

[2] S. I. Niculescu. Delay Effects on Stability: A Robust Control Approach. Springer-Verlag, Heidelberg, Germany, 2001.

[3] S. J. Chen and J. L. Lin. Robust stability of discrete time-delay uncertain singular systems. IEE Proc. Control Theory Appl., 151:4551, 2003.

[4] L. Dai. Singular Control Systems. Springer-Verlag, Berlin, 1989.
[5] B. Du, J. Lam, and Z. Shu. A delay-partitioning projection approach to stability analysis of neutral systems. In IFAC World Congress 2008, Seoul, 2008.

[6] B. Du, J. Lam, Z. Shu, and Z. Wang. A delay-partitioning projection approach to stability analysis of continuous systems with multiple delay components. IET Control Theory and Applications., 3:383-390, 2009.

[7] E. Fridman and U. Shaked. $H_{\infty}$ control of linear state-delay descriptor systems: an Imi approach. Linear Algebra \& Its Applications, 351352:271-302, 2002.

[8] H. Gao, T. Chen, and J. Lam. A new delay system approach to network based control. Automatica, 44:39-52, 2008.

[9] H. L. Gao, B. G. Xu, Y. G. Zhang, and X. L. Hou. A new delaydependent stability criterion for singular time-delay systems. In Proceedings of IEEE Conference on Control and Automation, pages 2252-2256, 2007.

[10] J. K. Hale and S. M. Verduyn Lunel. Introduction to Functional Differential Equations. Springer-Verlag, New York, 1993.

[11] X. Ji, H. Su, and J. Chu. Delay-dependent robust stability of uncertain discrete singular time-delay systems. In Proceedings of American Control Conference, pages 3843-3848, Minniapolis, Minneaota, USA, 2006.

[12] I. Petersen. A stabilization algorithm for a class of uncertain linear systems. Systems \& Control Letters, 8:351-357, 1987.

[13] R. L. Wang and Y. Q. Liu. Asymptotic stability and robustness for discrete-time singular systems with multiple time-delays. In Proceedings of 3th World Congress on Intelligent Control and Automation, Hefei, P.R, USA, 2000.

[14] Z. Wang, Y. R. Liu, and X. Liu. $H_{\infty}$ filtering for uncertain stochastic time-delay systems with sector-bounded nonlinearities. Automatica, 44:1268-1277, 2008.

[15] Z. Wu, H. Su, and J. Chu. Robust stabilization for uncertain discrete singular systems with state delay. Int. J. Robust \& Nonlinear Control, 18:1532-1550, 2008.

[16] S. Xu and J. Lam. Control and Filtering of Singular Systems. Springer, Berlin, 2006.

[17] S. Xu, J. Lam, and C. Yang. Robust $H_{\infty}$ control for discrete singular systems with state delay and parameter uncertainty. Dynamics Continuous Discrete Impul. Systems. Ser. B: Appl. Algorithms, 9:539554, 2002.

[18] S. Xu, J. Lam, and Y. Zhou. An improved characterization of bound realness for singular delay systems and its applications. Int. J. Systems Sci., 18:263-277, 2008.

[19] S. Xu, P. Van Dooren, R. Stefan, and J. Lam. Robust stability and stabilization for singular systems with state delay and parameter uncertainty. IEEE Trans. Automat. Control, 47:1122-1128, 2002.

[20] S. Zhu, Z. Cheng, and J. Feng. Delay-dependent robust stability criterion and robust stabilization for uncertain singular time-delay systems. In Proceedings of American Control Conference, Portland, OR, USA, 2005. 